\title{
Rapid adaptation to near extinction in microbial experimental evolution
}

\author{
Michael Travisano ${ }^{1,2}$ (D) Michihisa Maeda ${ }^{2,3} \cdot$ \\ Fumie Fuji ${ }^{2}$ Toshiaki Kudo ${ }^{2,4}$
}

(C) The Author(s) 2017. This article is an open access publication

\begin{abstract}
Theory indicates that responses to natural selection maximize immediate fitness benefits, leading to adaptations to current environmental conditions and those of the immediate past. Over a century of advances in theory, experiment, and observation have documented innumerable adaptations demonstrating the efficacy of natural selection to finely tune species to their respective environments. However, theory also suggests that natural selection is not a panacea, and that improvements in competitive ability do not necessarily increase long-term survival. Here we show that adaptation in experimental populations of microbes can dramatically reduce population sizes to near extinction levels in a stressful environment. The long-term potential for extinction differed from that identified in short-term ecological observations, but the eventual outcome is consistent with limitations on specific modes of adaptation. These results suggest that additional emphasis on the limitations of adaptation can provide insight on when and how improvements in competitive ability provide longerterm benefits.
\end{abstract}

Keywords Natural selection · Extinction · Competition

Michael Travisano

travisan@umn.edu

1 Department of Ecology, Evolution and Behavior, University of Minnesota, Saint Paul, MN 55108, USA

2 Institute of Physical and Chemical Research (RIKEN), WAKO, Saitama 351-01, Japan

3 School of Agriculture, Meiji University, Kawasaki, Kanagawa 214-8571, Japan

4 School of Marine Biosciences, Kitasato University, Sanriku, Ofunato, Iwate 022-0101, Japan 


\section{Introduction}

Adaptations are conspicuous aspects of biological systems. Understanding how a species is adapted to its environment remains a longstanding topic of investigation, because researchers continue to discover new and surprising adaptations (e.g. Tian et al. 2013). Individual adaptations can involve substantial biological complexity and the diversity of adaptations appears nearly inexhaustible. The perception of evolutionary outcomes derived solely from the abundance of extant adaptations might be boundless biological success, that species are likely to adapt successfully to even the most extreme environments. And yet, the vast majority of species over the long history of Earth have gone extinct (Raup 1994). While some of these extinctions involved external extreme perturbations to life (meteor strikes), which are necessarily be difficult to adapt to, such sudden, dramatic and simultaneous extinctions are not the norm (Payne and Finnegan 2007). Extinctions occur to species across of range of biological importance, from those that were ecologically dominant to those that were always numerically rare. But, there has been only modest success in incorporating observations of species loss and extinction into a general understanding of evolutionary processes (Gould 1989; Nixon and Wheeler 1992). Investigations into the causes of extinction are necessarily challenging because the species are typically already absent, or soon will be, and even studies on extinct species often focus on their unique adaptations, rather than the basis for their extinction (Forey and Chatterton 2003).

Recently, there is increasing interest in understanding the basis for extinctions, and their role in affecting biological function and diversity (Rankin and Lopez-Sepulcre 2005). Some of this interest involves recognition of an adaptationist bias in evolutionary biology, that has led to changes in null hypotheses and improved understanding of the limits of natural selection (Gould and Lewontin 1979; Pigluicci and Kaplan 2000). Two well known examples of adaptationist bias include misattribution of the basis of altruistic behaviors (Williams 1966) and a general expectation that the microbes in a microbiome are beneficial (Moran and Sloan 2015). A broader motivation for the increased interest is recognition of the dramatic environmental changes caused by human activity, which have persistent, widespread negative effects on available resources and habitats. Such changes disrupt living systems, including human populations. As environments become increasingly affected by human modification, pre-existing adaptations are mismatched to current habitats, so that many species are under threat of imminent extinction (Bellard et al. 2012). Environmental degradation is not unique to humans, as all species change their environment, typically drawing down resources and releasing waste products (Srivastava and Jefferies 1996). There have been numerous studies of adaptation to environmental change, but many of these studies suffer from an adaptationist perspective, so that understanding the limits of adaptation remains surprisingly thin (Bell 2012). Without such understanding, predictions regarding the generality and basis of successful adaptation are likely to be overly optimistic in forecasting the biological consequences of rapid environmental shifts.

To address these issues, we investigated the potential for adaptation under severe environmental conditions associated with habitat degradation. We made use of microbial experimental evolution to explore ecological and evolutionary outcomes (Lenski 
2017). Bacterial populations provide good model systems for the study of adaptation as they are relatively easy to culture and assay for growth. There is a wealth of information about the genetics, biochemistry and physiology of many bacterial species, and the biochemical pathways of stress responses are probably best known for bacterial species. We characterized the growth dynamics and evolutionary responses of bacteria under serial transfer, a periodically varying environment. In a serial transfer environment, the life history of a bacterial population can be described as four phases (Travisano 1997): (1) lag phase, the period of time required for bacteria to initiate growth after transfer to fresh medium, (2) growth phase, a period of exponential growth during which neither nutrient concentration nor waste products limit growth, (3) stationary phase, a period where reproduction does not occur due to nutrient limitation and/or build up of toxic waste metabolites, and (4) death phase, a period during which the population declines occur. Adaptation to periodic, serial transfer, conditions can involve improvements in one or more phases, such as shorter lag times or faster growth rates. Previous studies often focus primarily on exponential growth under ideal conditions, although there are exceptions (Fiegna and Velicer 2003).

The experiments presented in this paper were performed with the soil bacterium Alcaligenes xylosoxydans strain A41 during growth in a stressful environment, culture medium supplemented with biphenyl as the sole carbon and energy source. Biphenyl is a solid aromatic hydrocarbon, consisting of two benzene rings, that is hydrophobic, lipophilic and can readily diffuse into bacterial membranes. Biphenyl can be toxic to bacteria, even to those using it as a resource, due to its ability to disrupt cells and membranes (Sikkema et al. 1995) and the production of toxic metabolites during growth (Pérez-Pantoja et al. 2015). Much research into the ability of bacteria to grow on biphenyl is motivated by attempts to reduce pollution by polychlorinated biphenyls (PCBs), chlorinated analogues of biphenyl (Bedard et al. 1986). PCBs were previously widely used in electrical components due to their insulating ability and chemical stability, but their current use is greatly restricted because exposure is associated with numerous health issues (Kimbrough 1987). A. xylosoxydans A41 was isolated for its ability to degrade biphenyl (Ohta et al. 1996; Maeda et al. 1998), an ability that is not ubiquitous, but is found in a wide variety of unrelated bacterial species. In this study, the causes and effects of a stressful novel environment are examined. We addressed two related questions: How does the complexity of the seasonal environment affect the short term (ecological) and the longer term (evolutionary) likelihood for extinction? We show that short term ecological dynamics are predictable, that adaptation to the stressful conditions readily proceeds, but that the potential for extinction is enhanced as a consequence of adaptation.

\section{Boom and bust ecological cycles}

Upon transfer to fresh media supplemented with biphenyl, populations of A41 do not increase in population size immediately. Such growth dynamics are typical for bacteria, particularly in minimal nutrient media that does not contain complex amino acids, vitamins or other resources. Individual cells require time for the necessary components to be generated for cell division. Once population growth was initiated, 
Fig. 1 Irrespective of initial mortality or density, strain A41 experiences rapid exponential growth in $10 \mathrm{ml}$ medium containing $100 \mathrm{mg}$ biphenyl. Six cultures were assayed for each initial density, and confidence limits represent $95 \%$ confidence intervals

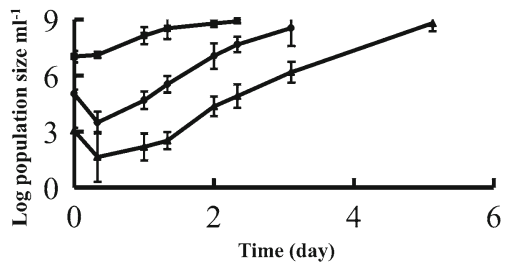

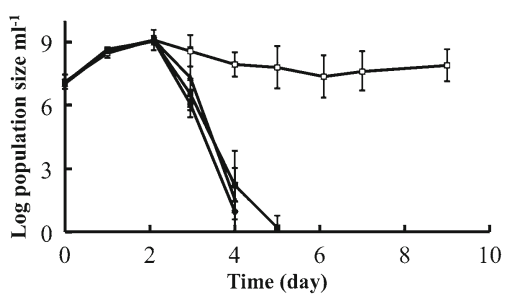

Fig. 2 Severe post-stationary phase mortality of A41 populations in $10 \mathrm{ml}$ medium depends upon the amount of initial biphenyl present. Death rate of the $100 \mathrm{mg}$ biphenyl treatment from 71 to $96 \mathrm{~h}$ is $-0.366 \pm$ 0.029. Five cultures were assayed for each treatment, and confidence limits represent $95 \%$ confidence intervals. (White circle) $10 \mathrm{mg}$ biphenyl; (black triangle) $30 \mathrm{mg}$ biphenyl; (black circle) $50 \mathrm{mg}$ biphenyl; (black square), $100 \mathrm{mg}$ biphenyl

experimental growth on biphenyl was observed, regardless of initial population density (Fig. 1) or amount of available biphenyl (data not shown). On $100 \mathrm{mg}$ biphenyl, we measured an exponential growth rate of $0.210 \pm 0.018 \mathrm{~h}^{-1}(95 \% \mathrm{CI})$, equivalent to a population doubling time of $\sim 3.3 \mathrm{~h}$, in a separate experiment with seven-fold replication. An essentially identical exponential growth rate was obtained for A41 grown in biphenyl-saturated medium containing no solid biphenyl $\left(0.204 \pm 0.036 \mathrm{~h}^{-1}\right.$; six replicates). These results demonstrate that strain A41 can grow rapidly in media with biphenyl as the sole carbon and energy source.

As the bacteria begin to exhaust resources and release waste products, growth slows and then ceases. In media containing $30 \mathrm{mg}$ or more biphenyl per $10 \mathrm{ml}$, A41 populations suffered exponential declines in viability after reaching population densities of $\sim 10^{9}$ cell ml $\mathrm{m}^{-1}$ (Fig. 2), and no viable cells were detected in these populations after day 4. Populations experienced little or no cell death after stationary phase in media having $10 \mathrm{mg}$ (or less, data not shown) of biphenyl. After 4 days, solid biphenyl remained only in those cultures initially containing $30 \mathrm{mg}$ or more biphenyl, but not in those containing $10 \mathrm{mg}$, suggesting that excess biphenyl was necessary for the mortality. No post-stationary phase cell death occurs in medium supplemented with other nutrients (data not shown).

Striking patterns of boom and bust population sizes were observed under different periodic transfer conditions. We examined the interaction of life history and stress on bacterial population dynamics, by transferring (100-fold dilution) populations of A41 in $100 \mathrm{mg}$ biphenyl per $10 \mathrm{ml}$ medium under different propagation schemes. Populations propagated daily slowly declined in density, even though the exponential growth rate is sufficient to make up for the daily dilution, because of the mortality which occurs after transfer to fresh medium during exponential growth (Fig. 3a). 


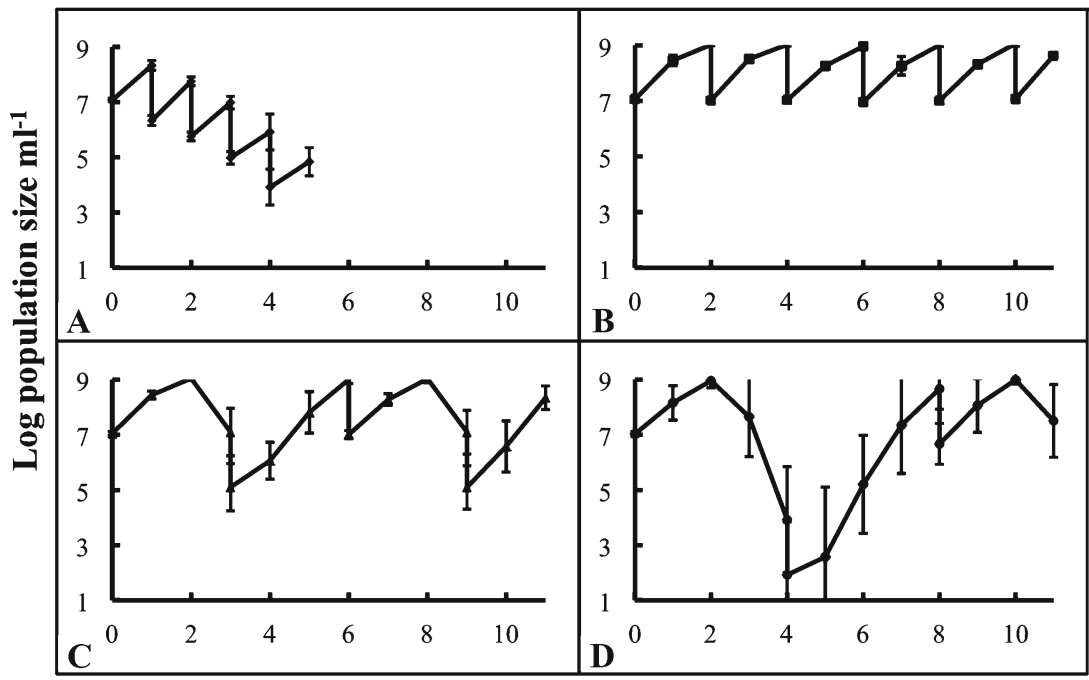

Time (day)

Fig. 3 Post-inoculation and post-stationary phase mortality has profound effects on population dynamics, depending upon the culture regime. A41 cultures in $10 \mathrm{ml} \mathrm{C}$-medium containing $100 \mathrm{mg}$ biphenyl were transferred by a 100-fold dilution: a every day; b every other day; c every third day; $\mathbf{d}$ every fourth day. Four cultures were assayed for each treatment, and confidence limits represent $95 \%$ confidence intervals

Populations propagated every other day had dynamics similar to those of classical bacterial serial culture (lag, exponential and stationary phases), giving no suggestion of cell mortality (Fig. 3b). Populations propagated every third (Fig. 3c) or every fourth (Fig. 3d) day experienced wide fluctuations in population size due to post-stationary phase mortality. These differences in population dynamics arose by changes in the period length of culturing: the number of days between transfers. If transfers are too frequent, populations cannot grow sufficiently rapidly to overcome the dilution and lag phase mortality. If the transfers are too infrequent, populations experiences wide swings in size that are prone to cause population extinctions, as expected from theory (Travisano 1997). These dynamics are partially driven by the growth of the bacteria themselves, as they exhaust the media and secrete waste products, with the 2-day transfer regime being best for consistent population growth.

\section{Short term evolutionary improvement but long term extinction}

Clearly there was substantial room for adaptation of the ancestral A41 genotype for growth on biphenyl. What was less certain was: (1) if the potential was realizable and (2) if short term ecological observations could inform patterns of evolutionary adaptation. These questions were addressed in a subsequent 60-day selection experiment. Replicate populations of A41 were propagated under one- and 2-day periodic environments with excess available biphenyl $(100 \mathrm{mg} / 10 \mathrm{~mL})$, and ten replicate populations per transfer regime. Each population underwent 100 -fold dilutions 


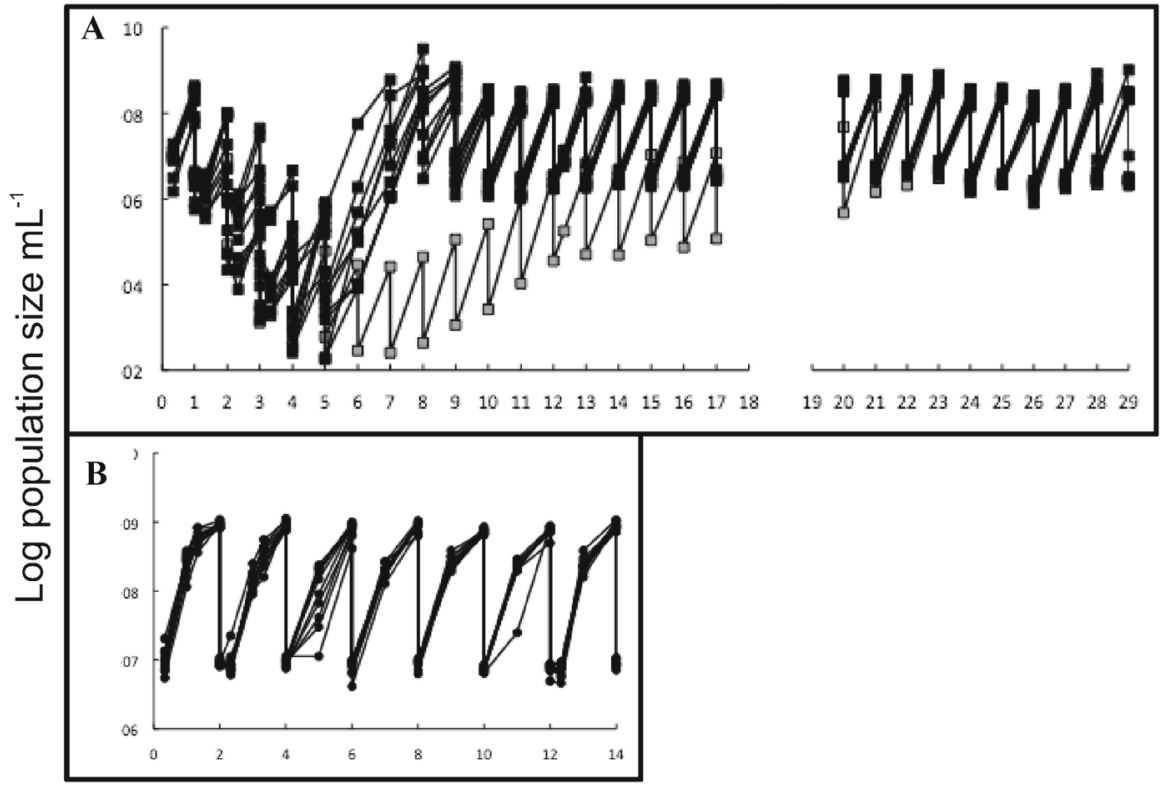

Fig. 4 Population sizes over time (days) of the replicate populations during selection with excess biphenyl, either on a one- (A) or 2-day (B) transfer regime. a Daily transfer was suspended for nine of ten populations on a 1-day transfer regime (black squares), when the populations were near extinction (between days 4 and 7). One population (grey squares) consistently showed some growth and was maintained on a 1-day regime through the experiment. Eventually, all the 1-day regime populations converged to the same population dynamics. b All of the 2-day regime populations reached the same maximal population density every other day during the first 2 days of selection. Additional platings on days 1-3 and 12 were performed to assay for reduced lag time for both one and 2-day transfer regimen populations, and are discussed in the main text

upon transfer. Population size for every replicate was estimated by plating on a daily basis for the first 14 days, to ensure that populations did not go extinct during the initial phase of the experiment, and then on a subset of timepoints for the remainder of the experiment. After transfer to fresh media, the culture tubes of the populations from the previous transfer were placed back into the incubator for observation. This was done to monitor growth of the old cultures over the subsequent $24 \mathrm{~h}$ and thereby determine if populations did have viable populations even if it initially appeared otherwise by the absence of culture turbidity.

Based upon the prior results, the populations transferred on a daily basis were expected to initially decline in density, which was observed (Fig. 4a). The steep declines in density across the daily transferred populations was also evident in the previous days culture tubes that had been returned to the incubator, after transferring the populations to fresh media. To avoid population extinctions, nine of the ten daily transferred populations were not transferred for 2-3 days (depending on the population) to allow for population recovery. One of the daily transferred population showed at least some growth in the previous days cultures tubes, and so was maintained on the daily cycle. This population slowly recovered density, despite the daily transfer regime (see Fig. 4a). Upon recovery, all daily propagated populations were able 
to maintain large population sizes throughout the remainder of the selection experiment. Additional evidence for adaptation was the ability of populations to rapidly begin growing upon transfer to fresh media, as can be seen by comparing the growth from days 1-3 with day twelve. After transfer to fresh media for the first 3 days of the selection, populations generally remained constant or declined in density during the first $8 \mathrm{~h}$, as can be seen by additional measurements made at the outset of the experiment. By day twelve, all populations increase in density, by an average of 3.6fold, during the first $8 \mathrm{~h}$ of growth (Fig. 4a). Their growth dynamics over the course of selection approached that of the ancestral genotype prior to selection, except on a 1-day transfer regime rather than the 2-day transfer required for stability at the outset of selection (Fig. 3b).

The replicate populations propagated on a 2-day transfer regime suffered no population declines during the initial two weeks of selection (Fig. 4b), in contrast to 1-day transfer populations (Fig. 4a). As expected from the ecological results obtained previously (Fig. 3b), the populations required 2 days become fully turbid and the population densities were initially stable on a 2 day cycle, prior to any apparent adaptations or changes in growth rate. No dramatic changes in initial growth rates (from 0 to $8 \mathrm{~h}$ ) were observed, comparing the populations after 2 and 12 days, in contrast with the 1-day transfer regime populations. This difference is not surprising. By 12 days, the 2-day populations had gone through fewer rounds of selection, and were being transferred at stationary phase, when cells are not actively replicating and require time to start regrowing. In contrast, the 1-day populations were growing exponentially, or nearly so, when transferred to fresh media by the twelfth transfer.

As the experiment progressed, the population dynamics of the 2-day replicate populations began to oscillate wildly, as was evident by culture turbidity (data not shown). The day following transfer, when the ancestral genotype had previously reached substantial population densities (Fig. 3b), one or more replicates would frequently have little if any evident bacterial growth. After the 60th day of bacterial culture, 30 transfers, the experiment was terminated when it was unclear if the experiment could be reliably continued due to the possibility of population extinctions. At that point, all of the replicates had undergone at least one near extinction event. Superficially at least, the population dynamics of the 2-day transfer populations had begun to resemble that of the ancestral genotype when propagated under a 3- and 4-day transfer regime, with frequent population crashes and complex population cycling (Fig. 3c, d).

\section{4 (Mal)-adaptation}

Increasing rates of population crashes and instability over the course of selection is a surprising outcome. This is especially true when population sizes are large and are not subject to fixation of deleterious mutations by genetic drift, as can occur in small populations (Nei et al. 1975). Moreover the apparent adaptation in the 1-day regime, strongly suggests genetic drift was not an important contributor to the evolutionary outcome in the 2-day transfer regime populations. We repeated our assays of bacterial population growth dynamics, but now with genotypes from the selected populations, at which point it became clear that adaptation to the selection regime was the basis for 


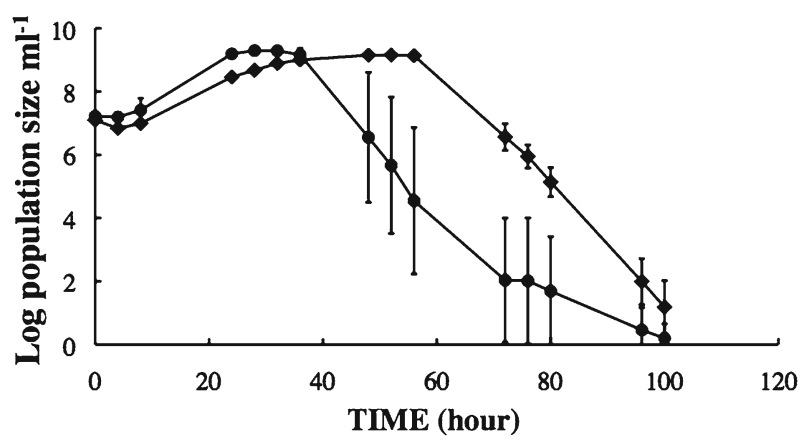

Fig. 5 Post-stationary phase mortality differs dramatically between the ancestral genotype (black diamond) and 2-day selected populations (circle) propagated on a 2-day regime. The values for the selected populations are the average of estimates for each of the ten populations after 60 days of selection (thirty transfers). Population estimates for the ancestor are the average of ten measurements for each time point. Error bars represent $95 \%$ confidence intervals, and are large for the selected populations because of differences among the selected populations, and not large errors of estimation (see Fig. 6)

the increased population instability. All populations, regardless of being transferred on a 1- or 2-day regime, had increased in growth rate and much more rapidly reached their maximal population density (Fig. 5 shows the 2-day growth dynamics for the 2-day regime populations). While the initial genotype required 2 days to reach maximal density, all of the selected populations required only a single day to reach the same density. For the populations maintained on a daily transfer regime cycle, this evolved phenotype was clearly beneficial, as the populations could be maintained stably through 60 days of selection (data not shown).

In contrast, increased growth rates were substantially more problematic for the populations transferred on a 2-day regime (Fig. 5). Within a population, genotypes with more rapid growth rates have a selective advantage to those with a slower growth rate. This is because total growth is limited by available resources and the concentration of waste metabolites in the culture media environment. Genotypes that grow faster produce more offspring than slower growing genotypes, and thereby displace them from the population. Over time, faster and faster growing genotypes become an increasingly large proportion of the population, so that maximal population size is reached earlier. Barring differences in death rate among the genotypes, populations adapt by growing faster and consequently dying earlier. The pattern of increasing growth rates and earlier onset of rapid population decline was observed in all ten replicate populations maintained on a 2-day transfer regime (Fig. 5).

One possible escape from population instability could have been evolution of resistance to the factors causing bacterial death after reaching maximal population size. As the 2-day populations were nearly extinct at every transfer by the end of the selection experiment, there did not appear to be much potential for such adaptation, nor direct methods to measure evolutionary responses. However, variation in death rates among the selected populations suggested that adaptive responses were, in theory, possible and had occurred during the selection experiment. We indirectly assessed this potential for adaptation to reduce death rates by comparing the growth and death rates of 


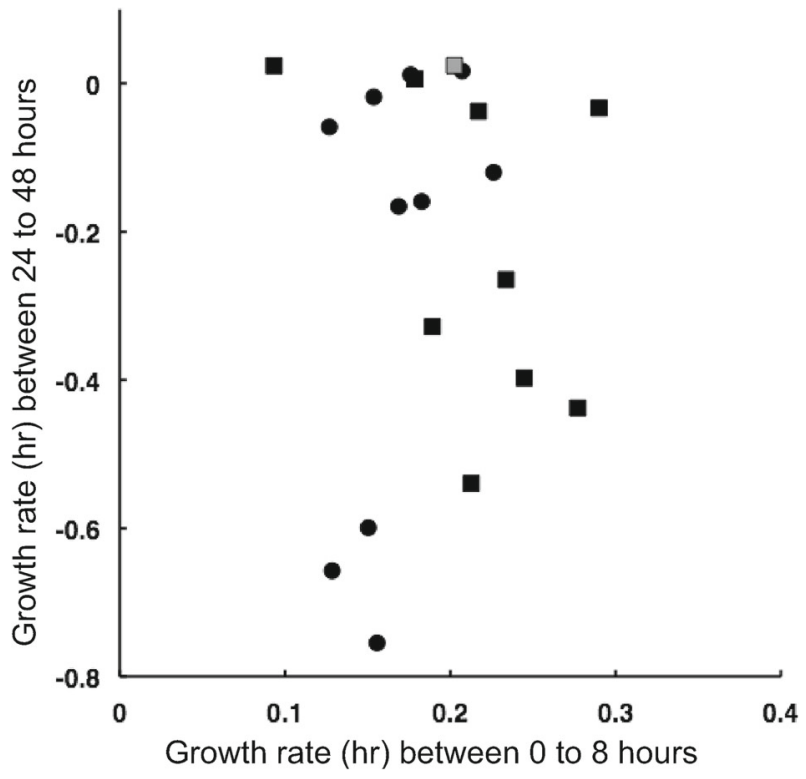

Fig. 6 Growth (and death) rates of 20 populations after thirty rounds of selection. Circles indicate populations transferred on a 2 days regime after 60 days, and squares indicate populations transferred on a daily regime for 30 days. Irrespective of transfer regime, there are no statistically significant differences in initial growth rate from 0 to $8 \mathrm{~h}$, nor death rate (= negative growth rate from 24 to $48 \mathrm{~h}$ ) The grey square is the single 1-day regime population maintained on a strict regime, without a transfer pause (see Fig. 4)

the 1- and 2-day selected populations. The 1-day populations had not experienced increased death rates during the course of selection over the first 30 days, but both sets of populations had been selected for more rapid growth. If the 2-day selected populations had selectively important, evolutionary responses to reduce death rates, then we could expect lower death rates in the 2-day populations (than the 1-day) and perhaps lower growth rates in the 2-day populations (than the 1-day), indicating a trade-off in growth and death rates (see Kerr et al. 2006 for similar reasoning). However, no differences between the two treatments was observed, nor was there any evidence for trade-offs either within or across treatments (Fig. 6). The 1- and 2-day selected populations did not differ, between groups, in either growth or death rates, suggesting that there was no response to selection to decreasing death in the 2-day transfer populations.

\section{Discussion}

Our results with simple bacterial populations maintained under harsh conditions demonstrate the challenges inherent in adaptation, and in our ability to predict modes of adaptation. Predictable ecological population dynamics were obtained that are largely in agreement with general theoretical predictions of population dynamics. In the short run, the ecological dynamics provided useful information on conse- 
quences of continued selection, but longer-term predictions were more challenging. As selection proceeded, increases in growth rate rescued populations from apparent impeding extinction in one treatment, but were leading to apparent extinction in the other treatment. At face value, these results indicate the highly contextual benefits of adaptation: the specific environment matters (Travisano and Lenski 1996; Satterwhite and Cooper 2015). The same adaptation can provide a benefit in one environment but substantial costs in similar, but different, environments. More worrying was the increasing potential for extinction as a consequence of adaptation, because of the dramatic declines in population size. The process of natural selection can facilitate the spread of adaptations that increase short-term fitness benefits, but these adaptations may not increase longer-term fitness, and can instead have substantial costs in survival (Matsuda and Abrams 1994; Webb 2003). Such outcomes belie optimistic perspectives of researchers that champion the power of competition to produce the optimal outcomes (see Maynard Smith 1978).

Even a cursory review of biological systems reveals adaptations. These adaptations are fascinating in the mechanisms involved, the diversity of attributes and their surprising intricacy (Driscoll and Travisano 2017). However, extant organisms and their adaptations are not an unbiased sampling of life, rather they are the currently successful representatives of a vastly larger number of extinct species. Most investigations in biological diversity focus on the basis for success, the adaptations, even for species for which there are no longer living representatives. This bias on success has the potential to blinker interpretation of the potential for competition, natural selection and adaptation to overcome challenges. Recognition of these biases has become increasingly essential because of the outsized role humans have in shaping the environment. Because humans have dominant effects on the environment, the survival and well-being of all living systems is directly affected by human activities, and relatively less on natural processes. Direct human interventions have dramatic effects on habitat, water and resource availability, causing precipitous losses in populations of many species and driving them to near extinction population levels. Indirect effects have caused systemwide changes in climate, marine environments and weather disturbances. The ability of species to ecologically accommodate or evolutionary adapt to these changes is far from guaranteed (Etterson and Shaw 2001). Rather, forecasts predict waves of species extinction. These challenges face human populations as well, because our direct dependence of products of biological systems and environmental system stability (Rockstrom et al. 2009).

Extrapolation from any study requires caution, and yet, our results suggest that there are opportunities for advancing understanding the role of competition more generally. In particular, the potential for competition to lead to undesirable outcomes in other systems, including some economic systems. While there are innumerable examples of the benefits of competition ultimately leading to functional improvements, in biological and economic systems, experimental investigation can increase understanding of competition and its benefits. Our current results suggest that a mismatch between short- and longer-term success can potentially lead to the collapse of the system itself. This conclusion illustrates the value of experimental evolution studies, which provide tractable experimental systems to develop proof of concept models (Zuk and Travisano, submitted). Using simplified experimental systems over 
evolutionary time, the causes and consequences for preferences (Marcus et al. 2018), diversity and competitive ability (Lensk and Burnham 2018) and system collapse can be disentangled (this study). The success of these studies demonstrates there are abundant opportunities for further advances.

Acknowledgements We thank T. Burnham, T. Dean, W. Driscoll, L. Forney, and R.E. Lenski for valuable discussions on this research, and an anonymous reviewer for many helpful suggestions. This work was supported by funded from the Japan Research Development Corporation and the U.S. National Science Foundation.

Open Access This article is distributed under the terms of the Creative Commons Attribution 4.0 International License (http://creativecommons.org/licenses/by/4.0/), which permits unrestricted use, distribution, and reproduction in any medium, provided you give appropriate credit to the original author(s) and the source, provide a link to the Creative Commons license, and indicate if changes were made.

\section{References}

Bedard, D. L., Unterman, R., Bopp, L. H., Brennan, M. J., Haberl, M., \& Johnson, C. (1986). Rapid assay for screening and characterizing microorganisms for the ability to degrade polychlorinated biphenyls. Applied Environmental Microbiology, 51, 761-768.

Bell, G. (2012). Evolutionary rescue and the limits of adaptation. Philosophical Transactions of the Royal Society of London. Series B, 368, 20120080.

Bellard, C., Bertelsmeier, C., Leadley, P., Thuiller, W., \& Courchamp, F. (2012). Impacts of climate change on the future of biodiversity. Ecology Letters, 15, 365-377.

Driscoll, W. W., \& Travisano, M. (2017). Synergistic cooperation promotes multicellular performance and unicellular free-rider persistence. Nature Communications, 15707.

Etterson, J. R., \& Shaw, R. G. (2001). Constraint to adaptive evolution in response to global warming. Science, 294, 151-154.

Fiegna, F., \& Velicer, G. J. (2003). Competitive fates of bacterial social parasites: persistence and selfinduced extinction of Myxococcus xanthus cheaters. Proceedings of the Royal Society B, 270, 15271534.

Forey, R., \& Chatterton, B. (2003). A Devonian trilobite with an eyeshade. Science, 301, 1689.

Gould, S. J. (1989). Wonderful life. New York, NY: W. W. Norton \& Co.

Gould, S. J., \& Lewontin, R. C. (1979). The spandrels of San Marco and the Panglossian paradigm: a critique of the adaptationist programme. Proceedings of the Royal Society B, 205, 581-598.

Kerr, B., Neuhauser, C., Bohannan, B. J., \& Dean, A. M. (2006). Local migration promotes competitive restraint in a host-pathogen "tragedy of the commons". Nature, 442, 75-78.

Kimbrough, R. D. (1987). Human health effects of polychlorinated biphenyls (PCBs) and polybrominated biphenyls (PBBs). Annual Review of Pharmacology and Toxicology, 27, 87-111.

Lenski, R. E. (2017). What is adaptation by natural selection? Perspectives of an experimental microbiologist. PLoS Genetics, 1006668.

Lenski, R. E. \& Burnham, T. C. (2018). Experimental evolution of bacteria across 60,000 generations, and what it might mean for economics and human decision-making. Journal of Bioeconomics, forthcoming.

Maeda, M., Roberts, M. S., Ohta, Y., Fuji, F., Travisano, M., \& Kudo, T. (1998). Isolation and characterization of a new aromatic compound-degrading alkalitrophic bacteria. Journal of General and Applied Microbiology, 44, 101-106.

Marcus, M., Burnham, T. C., Stephens, D. W., \& Dunlap, A. (2018). Experimental evolution of color preference for oviposition in Drosophila melanogaster. Journal of Bioeconomics, forthcoming.

Matsuda, H., \& Abrams, P. A. (1994). Runaway evolution to self-extinction under asymmetrical competition. Evolution, 48, 1764-1772.

Maynard Smith, J. (1978). Optimization theory in evolution. Annual Review of Ecology and Systematics, 9, 31-56.

Moran, N. A., \& Sloan, D. B. (2015). The hologenome concept: Helpful or hollow? PLoS Biology, 13, e1002311. 
Nei, M., Maruyama, T., \& Chakraborty, R. (1975). The bottleneck effect and genetic variability in populations. Evolution, 29, 1-10.

Nixon, K. C., \& Wheeler, Q. D. (1992). Extinction and the origin of species. In: Extinction and Phylogeny (pp. 119-143). Columbia University Press, New York.

Ohta, Y., Maeda, M., Kudo, T., \& Horikoshi, K. (1996). Isolation and characterization of solvent-tolerant bacteria which can degrade biphenyl/polychlorinated biphenyls. Journal of General and Applied Microbiology, 42, 349-354.

Payne, J1, \& Finnegan, S. (2007). The effect of geographic range on extinction risk during background and mass extinction. PNAS, 104, 10506-10511.

Pérez-Pantoja, D., Lieva-Novoa, P., Donoso, R. A., Little, C., Godoy, M., Pieper, D. H., \& González, B. (2015). Hierarchy of carbon source utilization in soil bacteria: Hegemonic preference for benzoate in complex aromatic compound mixtures degraded by Cupriavidus pinatubonensis strain JMP134. Applied and Environmental Microbiology, 81, 3914-3924.

Pigluicci, M., \& Kaplan, J. (2000). The fall and rise of Dr Pangloss: adaptationism and the Spandrels paper 20 years later. Trends in Ecology $\mathcal{E}$ Evolution, 15, 66-70.

Rankin, D. J., \& Lopez-Sepulcre, A. (2005). Can adaptation lead to extinction? Oikos, 111, 616-619.

Raup, D. M. (1994). The role of extinction in evolution. Proceedings of the National Academy of Sciences (USA), 91, 6758-6763.

Rockstrom, J., Steffen, W., Nonne, K., Persson, A., \& Chapin, I. I. I. F. S. (2009). A space operating space for humanity. Nature, 461, 472-475.

Satterwhite, R. S., \& Cooper, T. F. (2015). Constraints on adaptation of Escherichia coli to mixed-resource environments increase over time. Evolution, 69-8, 2067-2078.

Sikkema, J., de Bont, J. A., \& Poolman, B. (1995). Mechanisms of membrane toxicity of hydrocarbons. Microbiology and Molecular Biology Reviews, 59, 201-222.

Srivastava, D. S., \& Jefferies, R. L. (1996). A positive feedback: herbivory, plant growth, salinity, and the desertification of an Arctic salt-Marsh. Journal of Ecology, 84, 31-42.

Tian, X., Azpurua, J., Hine, C., Vaidya, A., Myakishev-Remple, M., Ablaeva, J., Zhiyong, M., Nevo, E., Gorbunova, V., \& Seluanov, A. (2013). High-molecular-mass hyaluronan mediates the cancer resistance of the naked mole rat. Nature, 499, 346-349.

Travisano, M. (1997). The effects of toxic metabolites on dynamics and fitness in laboratory populations. In Fukuda Horikoshi \& Kudo (Eds.), Microbial Diversity and Biodegradation (pp. 97-112). Tokyo: Japan Sci. Societies Press.

Travisano, M., \& Lenski, R. E. (1996). Long-term experimental evolution in Escherichia coli. IV. Targets of selection and the specificity of adaptation. Genetics, 143, 15-24.

Webb, C. (2003). A complete classification of Darwinian extinction in ecological interactions. American Naturalist, 161, 181-205.

Williams, G. C. (1966). Adaptation and Natural Selection. Princeton, NJ: Princeton University Press.

Zuk, M., \& Travisano. M. Models on the runway: How do we make replicas of the world? (submitted) 